\title{
The effects of self-report cognitive failures and cognitive load on antisaccade performance
}

\author{
Nick Berggren $^{1 *}$, Samuel B. Hutton ${ }^{2}$ and Nazanin Derakshan ${ }^{1}$ \\ 1 Department of Psychological Sciences, Birkbeck University of London, London, UK \\ ${ }^{2}$ Department of Psychology, University of Sussex, Brighton, UK
}

Edited by:

Gilles Pourtois, University of Ghent, Belgium

Reviewed by:

Raffael Kalisch, University Medical Center Hamburg-Eppendorf, Germany Valentina Rossi, Ghent University, Belgium

*Correspondence:

Nick Berggren, Affective and

Cognitive Control Lab, Department of Psychological Sciences, Birkbeck

University of London, Malet Street, London WC1E 7HX, UK.

e-mail: nbergg01@mail.bbk.ac.uk
Individuals reporting high levels of distractibility in everyday life show impaired performance in standard laboratory tasks measuring selective attention and inhibitory processes. Similarly, increasing cognitive load leads to more errors/distraction in a variety of cognitive tasks. How these two factors interact is currently unclear; highly distractible individuals may be affected more when their cognitive resources are taxed, or load may linearly affect performance for all individuals. We investigated the relationship between self-reported levels of cognitive failures (CF) in daily life and performance in the antisaccade task, a widely used tool examining attentional control. Levels of concurrent cognitive demand were manipulated using a secondary auditory discrimination task. We found that both levels of self-reported CF and task load increased antisaccade latencies while having no effect on prosaccade eye-movements. However individuals rating themselves as suffering few daily life distractions showed a comparable load cost to those who experience many. These findings suggest that the likelihood of distraction is governed by the addition of both internal susceptibility and the external current load placed on working memory.

Keywords: cognitive failures, antisaccade performance, distractibility, cognitive load

\section{INTRODUCTION}

Cognitive slips and errors are common in daily life, with most people at one time or another forgetting where they left their car keys or if they left a light switched on at home. However, some individuals are more likely to commit such slips than others. The cognitive failures questionnaire (CFQ; Broadbent et al., 1982) is an established measure of individual differences in daily cognitive slips, with a number of questions relating to everyday errors such as the likelihood of dropping objects or failing to keep a task goal in mind. While these kinds of questions might be seen to reflect lapses in memory, scores on this questionnaire are positively correlated with increased distraction of attention in a number of daily life situations: from absentmindedness while shopping (Reason and Lucas, 1984), to an increased number of car accidents (Larson and Merritt, 1991) and other mishaps or injuries at work (Wallace and Vodanovich, 2003). Notably, CFQ scores remain relatively constant over time (Broadbent et al., 1982), and spouse ratings consistently match self-reported scores (Hickox and Sunderland, 1992), demonstrating that CFQ is a robust index of daily life cognitive failures $(\mathrm{CF})$.

In addition to correlates with daily life slips, high CFQ scorers also show specific deficits in experimental investigations of attention and cognitive control. For instance, increased levels of CF are associated with increased interference in the Eriksen flanker task and Stroop task (Broadbent et al., 1986; Tipper and Baylis, 1987), and impaired performance when dividing attention (Harris and Wilkins, 1982). Tipper and Baylis (1987) also found that high CFQ scorers showed no evidence of negative priming or inhibition to distractors while consistent negative priming effects were seen for low scorers, suggesting that CF may be associated with a reduced ability to inhibit task-irrelevant information. Importantly, CFQ scores do not appear to predict performance in memory tasks (Wilkins and Baddeley, 1978), though they have been noted to affect memory in tasks requiring the inhibition of unwanted memories (Groome and Grant, 2005).

One explanation for the relationship between CFQ scores and poor performance on tasks of selective attention maybe that both reflect a failure to maintain task goals in working memory (WM). Loading WM via secondary tasks has been shown to disrupt selective attention in a similar manner to that reported in the study of CFQ (see, e.g., Gazzaley, 2011, for review). One paradigm exemplifying this is the antisaccade task, in which participants are required to inhibit a prosaccade toward a sudden onset target, and initiate a saccade toward its mirror image location. Converging evidence suggests that antisaccade performance is linked to WM processes. For example, participants with low WM span show increased errors (prosaccades toward the target; Unsworth et al., 2004). Increased errors and increased correct antisaccade latencies are found in populations with known WM deficits, such as elderly participants, and for first-episode schizophrenic patients (Nieuwenhuis et al., 2004; see Hutton and Ettinger, 2006, for review). In addition, secondary WM loads such as mental arithmetic or n-back correspondingly have adverse effects on correct antisaccade latencies and error rates (e.g., Roberts et al., 1994; Kane et al., 2001).

While both internal factors such as personality and external factors including cognitive load can adversely affect selective attention, very few investigations have attempted to examine the extent to which these two factors interact. In daily life, we are required to perform cognitive tasks that range in difficulty, in different 
environments that include varying sources of potential distractions, from a quiet office to driving on a busy road. Though one's susceptibility to CF and concurrent cognitive load are separate, in the real world they are not independent. Are individuals who experience little distraction in life more able to cope in situations where cognitive resources are taxed by load, and are those who experience many distractions less able to cope when situations are more cognitively demanding? Divided attention tasks provide some evidence that individuals reporting high levels of CF do suffer more distraction than low CF reporters, but these tasks tend not to compare performance across both single and dual-task conditions within the same experiment (e.g., Harris and Wilkins, 1982).

Accordingly, we investigated the role of both CFQ score and cognitive load using the antisaccade task. A secondary auditory task of either low or high load was also employed, requiring either passive verbal response or more complex pitch discrimination respectively. A previous investigation using the antisaccade task found that high CFQ score was correlated with faster antisaccade latencies but a greater number of errors (Larson and Perry, 1999). However, this finding might imply an unusual speed-accuracy trade-off in high CFQ scorers rather than evidence of a cognitive efficiency deficit, and the experiment also contained a number of other methodological concerns (see Discussion). We hypothesized that high levels of CF would be correlated with increased antisaccade latencies in line with previous findings involving groups characterized by deficits in cognitive inhibition (e.g., individuals with high levels of anxiety; Derakshan et al., 2009; see Derakshan and Eysenck, 2009, for a review). We also predicted that cognitive load would impair antisaccade latencies while not impacting upon prosaccade latencies. Finally, we examined the effect of load on both low and high CFQ scorers, while also measuring levels of state anxiety to ensure stressors did not affect scorers differently.

\section{MATERIALS AND METHODS PARTICIPANTS}

Forty-two participants (25 female) were recruited via advertisements at the University of London to take part in the experiment (mean age 24.36, range 20-36). Participants had normal or corrected-to-normal vision. They reported no auditory impairments.

\section{APPARATUS}

Eye-movements were recorded using an SR Research Eyelink 1000 eye-tracker (SR Research, ON, Canada).Only one eye was tracked during the experiment. Nine-point calibration across the computer screen was used to ensure tracking accuracy was within $1^{\circ}$ of visual angle. Images were presented on a $21^{\prime \prime}$ Viewsonic CRT monitor $(140 \mathrm{~Hz})$, and a chinrest was used to ensure a constant viewing distance of $60 \mathrm{~cm}$. The experiment was presented using the SR Research Experiment Builder software. A separate laptop played the auditory tones, presented through E-Prime software.

\section{STIMULI AND PROCEDURE}

The experiment took place in a dimly lit and sound-protected room. Prior to the experiment proper, participants completed the CFQ (Broadbent et al., 1982) in addition to the state version of the State-Trait Anxiety Inventory (Speilberger et al., 1983; also completed at the end of the experiment). In the main experiment, participants were instructed to look "AT," or "AWAY" from a white oval-shaped item appearing on-screen depending on the block, while also concurrently responding to tones presented in the background verbally (i.e., low or high load).

Each trial began with a fixation cross $\left(0.95^{\circ} \times 0.95^{\circ}\right)$ presented in the center of the screen for $1000 \mathrm{~ms}$. Participants were instructed to fixate this cross. If participants fixated the cross between 500 and $1000 \mathrm{~ms}$ after its onset, the trial moved forward immediately, acting as a drift correct to tracking. The oval-shaped target subtending $2.58^{\circ} \times 4.77^{\circ}$ then appeared either in the left or right periphery of the screen for $600 \mathrm{~ms}$, at an eccentricity from fixation to the center of the oval of $11.04^{\circ}$. In the prosaccade block, participants were asked to move their eyes from fixation to the target as quickly and as accurately as possible. In the antisaccade block, participants were instructed to move their eyes to the mirror image location as quickly and as accurately as possible, while trying to avoid looking toward the target. An inter-trial interval of $1500 \mathrm{~ms}$ then occurred.

For the secondary task, auditory tones were played in the background of the room via a laptop. In the high load condition, one of three tones differing in pitch was randomly presented every 1900-2300 ms (five choices of $100 \mathrm{~ms}$ increments). Participants were asked to respond with "low," "mid," or "high" depending on the pitch, while concurrently performing anti- and prosaccades. In the low load condition, participants were asked to simply say the word "tone" whenever one was played. Only the mid tones were used during this block, to ensure that participants did not implicitly discriminate the tone pitch despite not being required to. The experimenter informed the participant at the start of each block what combination of "AT"/"AWAY" and "TONE"/ "PITCH" they would be conducting. The experimenter started the tones at the same time as the participant pressed the escape key on the keyboard to begin the block, and stopped the auditory tones when a block ended. The experimenter monitored the participants' performance and prompted them if they made errors in the discrimination. Good speed and accuracy was emphasized for both tasks.

Participants were given initial practice at distinguishing the tones, along with 16 practice trials for anti/prosaccades. The main experiment consisted of 8 blocks of 36 trials (two blocks for each condition), and block order was counterbalanced across participants. Participants were thanked, debriefed, and paid $\mathfrak{E 5}$ for their contribution, at the end of the experiment.

\section{RESULTS}

The data for 34 participants were used in the analysis ${ }^{1}$. Trials in which no saccade was made, or trials in which a saccade was made in under $80 \mathrm{~ms}$ (anticipatory saccade; see Fischer et al., 1993) were excluded from analysis. This led to an average of $6.04 \%$ of trials being removed (no effects of CF group on percentage of trials removed were observed). Median CFQ score for the entire sample was $42(\mathrm{SD}=13.36)$. Participants were divided based on the median split as either low $(N=17)$ or high scorers $(N=17)$. The two groups' scores significantly differed from each other

${ }^{1}$ Data from eight participants were removed either for the percentage of excluded trials being above $40 \%$ or for error rates of over $50 \%$. 
$[$ Med $=30.41$ vs. $52.29 ; t(32)=8.47, p<0.001]$.Additionally, participants' self-reports of state anxiety before and after the experiment were averaged to obtain a composite single state anxiety score reflecting levels of state anxiety during the experimental session. In this respect mean state anxiety for the entire sample was 36.51 $(\mathrm{SD}=7.84)$.

\section{RESPONSE LATENCIES}

Response latency was analyzed only for correct trials. A 2 (Load: low, high) $\times 2$ (Task: Prosaccade, Antisaccade) $\times 2$ (Group: low CF, high CF) mixed ANOVA was conducted on median latencies, with the means of individual median scores reported herein. This revealed a trend for a main effect of Load $[F(1,32)=3.56$, $p=0.07]$ and a significant effect of Task $[F(1,32)=228.8$, $p<0.001]$. Participants were moderately slower under high load compared to low load (low load: $M=221, \mathrm{SD}=33$; high load: $M=228, \mathrm{SD}=38$ ), while also much slower on antisaccade $(M=270, \mathrm{SD}=47)$ vs. prosaccade trials $(M=178, \mathrm{SD}=29)$. Importantly, there was a significant Load $\times$ Task interaction $[F(1,32)=9.75, p<0.005]$ with load significantly increasing antisaccade response latencies $[t(33)=3.48, p=0.001$; low load: $M=262, \mathrm{SD}=48$; high load: $M=279, \mathrm{SD}=51]$, while having no effect on prosaccade latencies ( $t<1$; low load: $M=179, \mathrm{SD}=31$; high load: $M=176, \mathrm{SD}=33$ ).

\section{Effects of CFO scores and state anxiety}

While no main effect of Group was observed $[F(1,32)=2.2$, $p=0.15]$, a significant Task $\times$ Group interaction $[F(1,32)=4.08$, $p=0.05$ ] was found. To assess this relationship further, we employed correlational analysis on each condition using CFQ score as a continuous variable. There was a positive relationship between CFQ scores and antisaccade latencies, $r=0.411, p=0.01$ (see Figure 1), and this relationship was significant for both low $(r=0.439, p<0.01)$ and high cognitive load $(r=0.352, p<0.05)$ conditions. The two correlation coefficients did not differ significantly from each other $(Z=0.41, p=0.68)$ Meanwhile, no significant correlation was found with prosaccade latencies under low, $r=0.03$, or high cognitive load, $r=0.13$, $p$ 's $>0.1$. Finally, Group did not significantly interact with Load or Load and Task together $(F$ 's $<1)$; load cost specifically on antisaccade latencies was comparable when examining CF groups separately (low CF: $M$ diff $=26 \mathrm{~ms}, \mathrm{SD}=35$; high $\mathrm{CF}: M$ diff $=14 \mathrm{~ms}, \mathrm{SD}=39)$.

Self-reported state anxiety correlated with CFQ scores ( $r=0.397, p<0.03$; see Figure 2), while showing a moderate relationship also with antisaccade latencies $(r=0.33, p=0.055)$. A hierarchical regression analysis was performed with CFQ scores entered on Step 1 and state anxiety on Step 2, to examine if state anxiety explained additional variance in antisaccade latencies after allowing for the main contributing effect of CFQ scores. State anxiety did not significantly predict AS latencies (unstandardized $\beta=1.19$, SE $\beta=1.04, t=1.14, p=0.24$ ), after allowing for the effect of CFQ scores (unstandardized $\beta=1.44$, SE $\beta=0.56$, $t=2.55, p<0.02$ ).

\section{ERROR RATES}

Errors were defined as saccades either to the oval target when participants were instructed to look away, or saccades away from

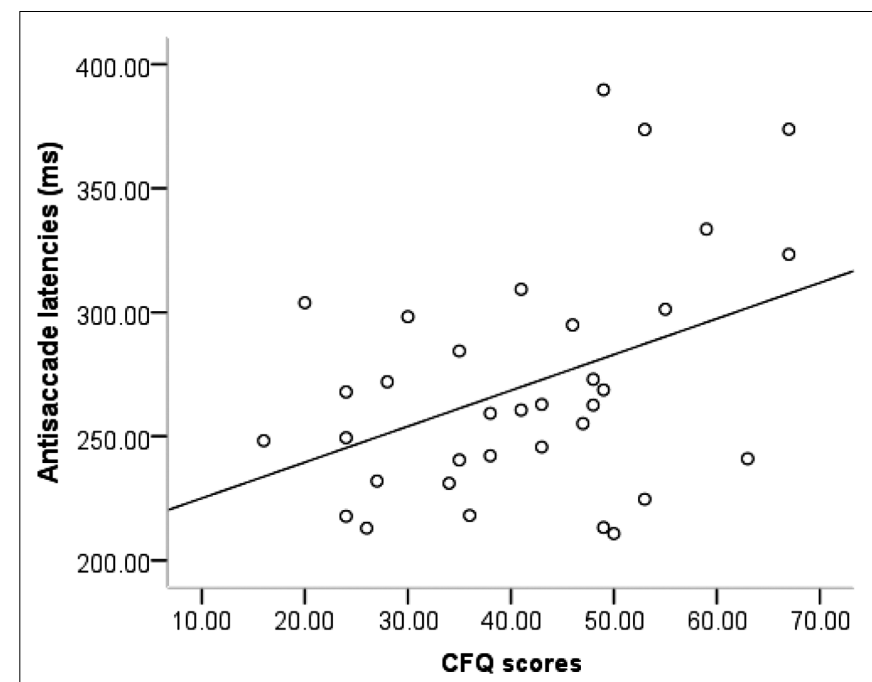

FIGURE 1 | Relationship between CFO scores and antisaccade latencies (collapsed across load).

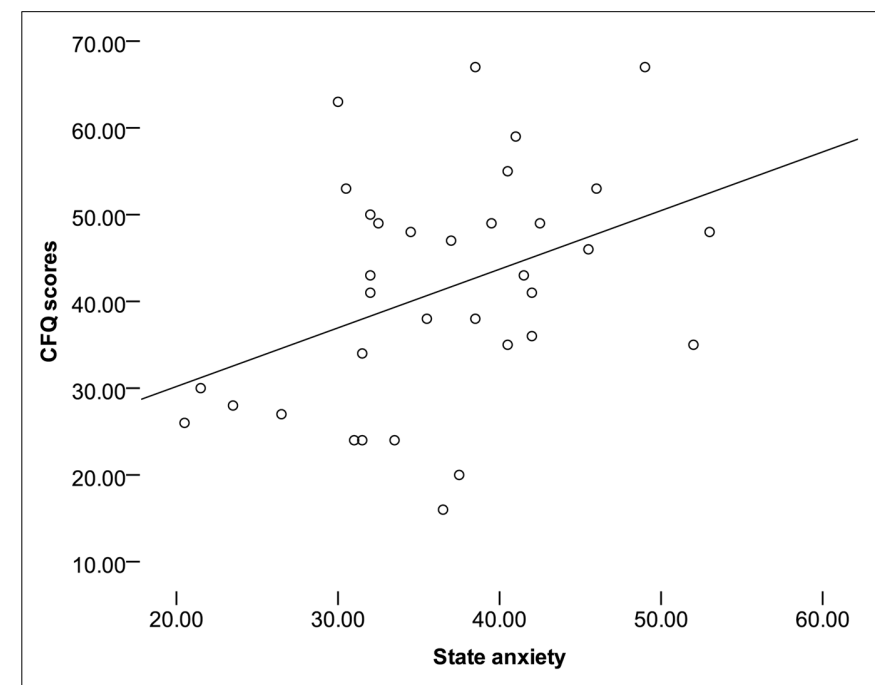

FIGURE 2 | Relationship between CFO scores and state anxiety.

the target when instructed to look at it. A 2 (Task; Antisaccade, Prosaccade $) \times($ Load: low, high $) \times 2$ (Group: low CF, high $\mathrm{CF}$ ) mixed ANOVA assessed reflexive errors (antisaccade trials) and possible incorrect inhibition (prosaccade trials) as percentages of the total number of trials. Analysis revealed a main effect of Load, $F(1,32)=88.92, p<0.001$, and a main effect of Task, $F(1,32)=4.7, p<0.05$. Participants made more errors under high load $(M=13.99, \mathrm{SD}=7.69)$ compared to low load $(M=11.62$, $\mathrm{SD}=7.71)$ and, as would be expected, far more errors were made on antisaccade trials $(M=19.93, \mathrm{SD}=10.62)$ vs. prosaccade trials $(M=5.68, \mathrm{SD}=4.83)$. The Task $\times$ Load interaction was not significant $(F<1)$.

There was no main effect of Group or interaction with Load $(F$ 's $<1)$. Furthermore, Task $\times$ Group did not interact 
$[F(1,32)=1.57, p>0.2]$, and there was no three-way interaction $[F(1,32)=1.68, p>0.2]$. Scores or state anxiety correlated with errors committed under high or low load in either anti- or prosaccade tasks (all $r$ 's $<0.1$ ).

\section{DISCUSSION}

The present study establishes that both one's dispositional susceptibility to CF and the situational cognitive load imposed on task goals can additively increase the likelihood of distraction as assessed by the antisaccade task. Both CFQ score and load caused clear detrimental effects on antisaccade latency, while having no effect on prosaccades, thus demonstrating that both measures purely affected trials requiring inhibition. Importantly, we found no evidence of a differential effect of load for low and high CFQ scorers on latencies and this would suggest an additive, rather than interactive, role of both these factors in distraction.

Our findings build upon previous research in a number of ways. To our knowledge, only one previous study has documented a relationship between CFQ score and lapses in overt attention (Larson and Perry, 1999). Establishing this point is important considering that many previous laboratory investigations of CFQ score do not always present visual displays for periods that preclude eye-movements. Differences in overt attention might therefore account for increased distractor processing in high CFQ scorers. Additionally, this previous study on CFQ score and overt attention had a number of methodological concerns, the most pressing being long eye-movement latencies within a normal population of over $480 \mathrm{~ms}$ on average for a reflexive prosaccade. Unusually, this average is far longer than reported here or even for latencies reported in clinical populations (e.g., Gooding and Tallent, 2001). Moreover, the experiment had no counterbalancing of block order, with prosaccade blocks always first, and this could potentially interact with individual differences in the latter condition (see Kane et al., 2001, for comment). Here, we distinguished between trials that were characterized by no saccades, anticipatory saccades, and erroneous reflexive saccades, establishing that CFQ only affected correct antisaccade latencies as opposed to reflexive errors. This finding is comparable to other studies investigating antisaccade differences within the general population (e.g., trait anxiety; Derakshan et al., 2009), and contests the point that CFQ is necessarily associated with overt attention as we did not observe any effects on error rates. In other words, high CFQ scorers were merely slower to disengage from the target on an antisaccade trial, which possibly only reflects a lapse in covert attention.

Additionally, our manipulation of load also slowed antisaccade latencies while having no effect on prosaccade latencies. Previous manipulations of load in overt attention tasks have relied upon concurrent n-back (Mitchell et al., 2002), mental arithmetic (Roberts et al., 1994), or concurrent tapping at set intervals (Stuyven et al., 2000). Roberts et al. (1994) presented numbers which participants were required to add together mentally in sets of five items, and so it is somewhat difficult to operationalize effects of load as every five sequences a load began from zero, while additionally numbers were given at no set interval by the experimenter vocally, creating a possible experimenter bias in sequence timing in the low load (repeat the number spoken by the experimenter) and high load (add each number spoken) conditions. Tapping load also has potential issues with participants timing saccades to keypress metronome, evidenced by the finding that tapping increases prosaccade as well as antisaccade latencies (Stuyven et al., 2000). Here, our load manipulation appears a useful tool for future research, avoiding the issue of keypresses by using auditory responses. That said, our method of monitoring participants' accuracy in the secondary task, with the experimenter prompting volunteers when appropriate, could be improved upon in future research, with the use of more quantitative methods registering participant responses. It should be noted however that our pitch discrimination of low, mid, and high tones may have been an effective load because it involved a spatial aspect of pitches relative to one another. Indeed, n-back load also requires an understanding of the spatial order of items, and so it is possible that cognitive load only affects antisaccade and overt attention performance when that load taxes the same sub-component of WM needed to perform a task (see Kim et al., 2005, for a similar argument on covert attention).

The finding that load did not affect CFQ scorers differently is particularly important to our understanding of daily life distraction. As mentioned, if anything high CFQ scorers showed a smaller increase in latencies under load than low scorers. That said, CFQ scores significantly predicted slower response latencies under both low and high load alike. We suggest that CF may cause an increased likelihood of lapses in attention, but that cognitive load simply adds to this susceptibility by linearly decreasing cognitive resources for all individuals equally. Considering that a link between CFQ score and WM capacity has not been established in previous studies, our findings suggest that CFQ score may be associated with deficits in cognitive efficiency (i.e., inhibition) rather than a reduced capacity. Hence, cognitive load would act by reducing the amount of available cognitive resources, but would not interact with a factor like CFQ score, which instead reduces the efficiency of cognitive resources in suppressing task-irrelevant information.

The present study also measured levels of state anxiety, due to conceptual concerns that state experiences of worry, and anxiety during a demanding cognitive task could explain any observed differences between low and high CF scorers. In contrast, our evidence suggested that although state anxiety was associated with antisaccade latency performance, CFQ was incrementally a much strong predictor on performance. Furthermore, state anxiety did not predict performance when the influence of CFQ score was taken into account. Thus, our findings suggest that anxiety during a difficult cognitive task can predict performance, but this anxiety is inherently explained by one's self-perceptions of distractibility. It should be noted that we did not assess the relationship between trait anxiety (sustained personality characteristics of anxiety) on CFQ score's predictive power. Previous work has shown that high trait anxious individuals exhibit larger costs on performance under high cognitive load (Berggren et al., in press), and also that trait anxiety score and CFQ score are positively correlated (e.g., Smith et al., 1995). Thus, one might argue that trait anxiety could account for some of the variance explained by CFQ score, despite our evidence suggesting that CFQ scorers are no differently affected in magnitude by load. Future research would benefit from 
more directly comparing these two personality factors together in affecting lapses in overt attention.

As well as that high CFQ scorers did not show greater costs on performance as load increased, it is also interesting to interpret results that low scorers, while under both low and high load faster than high scorers, did not show any reduced cost by cognitive load. This finding has relevance to the study of individual risk perception in the context of increasingly demanding daily tasks. White et al. (2004), for example, showed that while drivers agreed that concurrently using a mobile phone was one of the riskiest activities one could engage in, they perceived their own personal risk of an accident by doing this as less likely than for other people. This optimism bias is clearly not supported in the present study, where the addition of a demanding dual-task impaired performance at a similar magnitude both for individuals who perceive themselves as making few CF in daily life, and those who consider themselves

\section{REFERENCES}

Berggren, N., Koster, E. H., and Derakshan, N. (in press). The effect of cognitive load on emotional attention and trait anxiety: an eye-movement study. J. Cogn. Psychol.

Broadbent, D. E., Broadbent, M. H. P., and Jones, J. L. (1986). Performance correlates to self-reported cognitive failure and of obsessionality. $\mathrm{Br}$. J. Clin. Psychol. 25, 285-299.

Broadbent, D. E., Copper, P. F., FitzGerald, P., and Parkes, K. R. (1982). The cognitive failures questionnaire (CFQ) and its correlates. Br. J. Clin. Psychol. 21, 1-16.

Derakshan, N., Ansari, T. L., Hansard, M., Shoker, L., and Eysenck, M. W. (2009). Anxiety, inhibition, efficiency, and effectiveness: an investigation using the antisaccade task. Exp. Psychol. 56, 48-55.

Derakshan, N., and Eysenck, M. W. (2009). Anxiety, processing efficiency and cognitive performance: new developments from attentional control theory. Eur. Psychol. 14, 168-176.

Fischer, B., Weber, H., Biscali, M., Aiple, F., Otto, P., and Stuhr, V. (1993). Separate populations of visually guided saccades in humans: reaction times and amplitudes. Exp. Brain Res. 92, 528-541.

Gazzaley, A. (2011). Influence of early attentional modulation on working memory. Neuropsychologia 49, 1410-1424.

Gooding, D. C., and Tallent, K. A. (2001). The association between antisaccade task and working memory task performance in schizophrenia and bipolar disorder. J. Nerv. Ment. Dis. 189, 8-16.

Groome, D., and Grant, N. (2005). Retrieval-induced forgetting is inversely related to everyday cognitive failures. Br. J. Psychol. 96, 313-319.

Harris, J. E., and Wilkins, A. J. (1982). Remembering to do things: a theoretical framework and an illustrative experiment. Hum. Learn. 1, 123-136.

Hickox, A., and Sunderland, A. (1992). "Questionnaire and checklist approaches to assessment of everyday memory problems," in A Handbook of Neuropsychological Assessment, eds J. R. Crawford, D. M. Parker, and W. W. McKinlay (Hove: Erlbaum), 103-113.

Hutton, S. B., and Ettinger, U. (2006). The antisaccade task as a research tool in psychopathology: a critical review. Psychophysiology 43, 302-313.

Kane, M. J., Bleckley, M. K., Conway, A. R. A., and Engle, R. W. (2001). A controlled-attention view of working-memory capacity. J. Exp. Psychol. Gen. 130, 169-183.

Kim, S. Y., Kim, M. S., and Chun, M. M. (2005). Concurrent working memory load can reduce distraction. Proc. Natl. Acad. Sci. U.S.A. 102, 16524-16529.

Larson, G. E., and Merritt, C. R. (1991). Can accidents be predicted? An empirical test of the cognitive failures questionnaire. Appl. Psychol. Int. Rev. 40, 37-45.

Larson, G. E., and Perry, Z. A. (1999). Visual capture and human error. Appl. Cogn. Psychol. 13, 227-236.

Mitchell, J. P., Macrae, C. N., and Gilchrist, I. D. (2002). Working memory and the suppression of reflexive saccades. J. Cogn. Neurosci. 14, 95-103.

Nieuwenhuis, S., Broerse, A., Nielen, M. M. A., and de Jong, R. (2004). A

to commit many. An interesting avenue of future research would be to examine more closely the relationship between individuals' risk perception and their ability to perform a task under varying cognitive demands, such as within a driving simulator.

In summary, both self-reported CF in daily life and cognitive load predict performance in overt attention and the ability to ignore distraction. The contributions of both these internal and external factors appear to cumulatively govern the likelihood of focused attention and cognitive efficiency.

\section{ACKNOWLEDGMENTS}

This research was carried out by Nick Berggren under the supervision of Nazanin Derakshan at the Affective and Cognitive Control Lab in the Department of Psychological Sciences at Birkbeck University of London. NB is supported by a $1+3$ ESRC Ph.D. studentship.

goal activation approach to the study of executive function: an application to antisaccade tasks. Brain Cogn. 56, 198-214.

Reason, J. T., and Lucas, D. (1984). Absent-mindedness in shops: its incidence, correlates and consequences. Br. J. Clin. Psychol. 23, 121-131.

Roberts, R. J., Hager, L. D., and Heron, C. (1994). Prefrontal cognitive processes: working memory and inhibition in the antisaccade task. J. Exp. Psychol. Gen. 123, 374-393.

Smith, A., Chappelow, J., and Belyavin, A. (1995). Cognitive failures, focused attention, and categoric search. Appl. Cogn. Psychol. 9, S115-S126.

Speilberger, C. C., Gorsuch, R. L., Lushene, R., Vagg, P. R., and Jacobs, G. A. (1983). Manual for the State-Trait Anxiety Inventory. Palo Alto, CA: Consulting Psychologists Press.

Stuyven, E., Van der Goten, K. Vandierendonck, A., Claeys, K., and Crevits, L. (2000). The effect of cognitive load on saccadic eye movements. Acta Psychol. (Amst.) 104, 69-85.

Tipper, S. P., and Baylis, G. C. (1987) Individual differences in selective attention: the relation of priming and interference to cognitive failure. Pers. Individ. Dif. 8, 667-675.

Unsworth, N., Schrock, J. C., and Engle, R. W. (2004). Working memory capacity and the antisaccade task: individual differences in voluntary saccade control. J. Exp. Psychol. Learn. Mem. Cogn. 30, 1302-1321.

Wallace, J. C., and Vodanovich, S. J. (2003). Can accidents and industrial mishaps be predicted? Further investigation into the relationship between cognitive failure and reports of accidents. J. Bus. Psychol. 17, 503-514.

White, M. P., Eiser, J. R., and Harris, P. R. (2004). Risk perceptions of mobile phone use while driving. Risk Anal. 24, 323-334.

Wilkins, A. J., and Baddeley, A. D. (1978). "Remembering to recall in everyday life: an approach to absent-mindedness," in Practical Aspects of Memory, eds M. M. Fruneberg, P. E. Morris, and R. N. Sykes (London: Academic Press), 27-34.

Conflict of Interest Statement: The authors declare that the research was conducted in the absence of any commercial or financial relationships that could be construed as a potential conflict of interest.

Received: 01 August 2011; accepted: 05 October 2011; published online: 25 October 2011.

Citation: Berggren N, Hutton SB and Derakshan $N$ (2011) The effects of self-report cognitive failures and cognitive load on antisaccade performance. Front. Psychology 2:280. doi: 10.3389/fpsyg.2011.00280

This article was submitted to Frontiers in Cognition, a specialty of Frontiers in Psychology.

Copyright (C) 2011 Berggren, Hutton and Derakshan. This is an open-access article subject to a non-exclusive license between the authors and Frontiers Media $S A$, which permits use, distribution and reproduction in other forums, provided the original authors and source are credited and other Frontiers conditions are complied with. 